\title{
ASO Author Reflections: Is Robot-Assisted Radical Cystectomy with Intracorporeal Urinary Diversion the Way Forward?
}

\author{
Jeremy Yuen-Chun Teoh, FRCSEd (Urol) ${ }^{1,2}$, and Eddie Shu-Yin Chan, FRCSEd (Urol) ${ }^{1}$ \\ ${ }^{1}$ Department of Surgery, S.H. Ho Urology Centre, The Chinese University of Hong Kong, Hong Kong, China; ${ }^{2}$ European \\ Association of Urology Young Academic Urologists, Urothelial Carcinoma Working Group (EAU-YAU), Arnhem, \\ Netherlands
}

\section{PAST}

Robot-assisted radical cystectomy (RARC) has gained increasing interest in the past decade in treating patients with muscle-invasive bladder cancer (MIBC). ${ }^{1}$ However, even with the use of the robotic system, intracorporeal urinary diversion (ICUD) is still technically challenging, and it may be difficult to adopt. Whether RARC with ICUD confers any perioperative benefits when compared with extracorporeal urinary diversion (ECUD) is unknown.

\section{PRESENT}

The Asian RARC consortium has been established since 2017, aiming to investigate the potential benefits and harms of RARC from real-world experiences. In this study, ${ }^{2}$ bladder cancer patients undergoing RARC were included, and we compared the ICUD and ECUD groups. Our analysis showed that the ICUD group had less estimated blood loss and a shorter hospital stay than the ECUD group; ICUD remained as a significant protective factor upon multivariate analysis. Of note, both the ICUD and ECUD groups had similar rates of complications, be they minor or major in nature. Upon multivariate analysis, ICUD was also not associated with any increased risk of complications. We concluded that RARC with ICUD was safe and technically feasible to perform, with potential benefits of reduced blood loss and a shorter hospitalization.

\section{(C) Society of Surgical Oncology 2021}

First Received: 11 June 2021

Accepted: 11 June 2021;

Published Online: 24 June 2021

J. Y.-C. Teoh, FRCSEd (Urol)

e-mail: jeremyteoh@surgery.cuhk.edu.hk

\section{FUTURE}

RARC with ICUD is possibly one of the most minimally invasive surgical approaches in treating MIBC. While the procedure itself might be technically demanding, it is certainly generalizable in a multicenter setting as reflected by our study. ${ }^{2}$ More high-quality trials addressing this are urgently needed. The iROC study is multicenter randomized trial comparing RARC plus ICUD and open radical cystectomy. ${ }^{3}$ The initial results showed that RARC with ICUD was safe and well tolerated by the patients. ${ }^{4}$ The final results are eagerly awaited, and perhaps one day, RARC with ICUD can become the standard of care in treating localized MIBC.

DISCLOSURE The authors declare no conflicts of interest.

\section{REFERENCES}

1. Yee $\mathrm{CH}$, Yuen-Chun J, Chan ES. Current evidence for robotic surgery in radical cystectomy. Turk J Urol. 2021;47(Supp. 1):S1-8. https://doi.org/10.5152/tud.2020.20355.

2. Teoh JY, Chan E, Kang SH, et al. Perioperative outcomes of robotassisted radical cystectomy with intracorporeal versus extracorporeal urinary diversion. Ann Surg Oncol. 2021. https://doi.org/10. 1245/s10434-021-10295-5.

3. Catto JWF, Khetrapal P, Ambler G, et al. Robot-assisted radical cystectomy with intracorporeal urinary diversion versus open radical cystectomy (iROC): protocol for a randomised controlled trial with internal feasibility study. BMJ Open. 2018;8(8):e020500. https://doi.org/10.1136/bmjopen-2017-020500.

4. Catto JWF, Khetrapal P, Ambler G, et al. Multidomain quantitative recovery following radical cystectomy for patients within the robot-assisted radical cystectomy with intracorporeal urinary diversion versus open radical cystectomy randomised controlled trial: the first 30 patients. Eur Urol. 2018;74(4):531-4. https://doi. org/10.1016/j.eururo.2018.05.008.

Publisher's Note Springer Nature remains neutral with regard to jurisdictional claims in published maps and institutional affiliations. 